\title{
The effect of prehospital epinephrine in out-of-hospital cardiac arrest: a systematic review and meta-analysis
}

\author{
Authors: Wan Y Teoh ${ }^{\mathrm{A}}$ and $\mathrm{KaT} \mathrm{Ng}{ }^{\mathrm{B}}$
}

\section{Introduction}

Epinephrine has been recommended for out-of-hospital cardiac arrest (OHCA) resuscitation for nearly a century, but its efficacy and safety remain unclear in the literature. The primary aim of this review was to determine whether epinephrine increases the return of spontaneous circulation in OHCA patients.

\section{Method}

We conducted a systematic review and meta-analysis using the following databases: MEDLINE, EMBASE and CENTRAL from their inception until October 2018. All the randomised controlled trials (RCTs) were included. Observational studies, case reports, case series and non-systematic reviews were excluded.

\section{Main results}

Two trials including 8,548 patients were eligible for inclusion in the data synthesis. In patients who received epinephrine during OHCA, the incidence of return of spontaneous circulation was increased, with an odds ratio (95\% confidence interval $(\mathrm{Cl})$ ) of 4.25 (3.79-4.75), $p<0.001$, high quality of evidence. The number of patients transported to hospital was increased in patients who had prehospital epinephrine, with an odds ratio $(95 \% \mathrm{Cl})$ of 2.31 (2.11-2.53), $p<0.001$, high quality of evidence. The prehospital use of epinephrine was associated with an increased survival to hospital discharge, the odds ratio $(95 \% \mathrm{Cl}$ ) being 1.43 (1.10-1.87), $p=0.008$, moderate quality of evidence. No significant effect was noted on the favourable neurologic state of patient at hospital discharge, with an odds ratio $(95 \% \mathrm{Cl})$ of $1.21(0.90-1.64), p=0.21$, moderate quality of evidence.

\section{Conclusions}

In summary, this meta-analysis suggests that the prehospital use of epinephrine increases return of spontaneous circulation, transport of patients to hospital and survival to hospital discharge for OHCA. However, no significant effects on favourable neurologic function at hospital discharge were demonstrated. The general quality of evidence ranged from moderate to high.

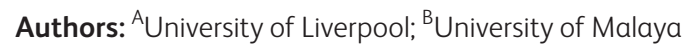

